\title{
ESTRATÉGIAS IMPLEMENTADAS EM HEMOCENTROS PARA AUMENTO DA DOAÇÃO DE SANGUE
}

\author{
Strategies implemented in hemotherapy services to increase blood donation \\ Estrategias incorporadas en hemocentros para el incremento de la donación de sangre
}

\author{
Leticia Carlesso \\ Universidade Federal de Santa Maria - UFSM - Santa Maria (RS) - Brasil
}

\section{Rosane de Fátima da Silva Guimarães}

Universidade Federal de Santa Maria - UFSM - Santa Maria (RS) - Brasil

\section{Suzel Lima da Silva}

Universidade Federal de Santa Maria - UFSM - Santa Maria (RS) - Brasil

Cristiane Ferreira dos Santos

Universidade Federal de Santa Maria - UFSM - Santa Maria (RS) - Brasil

Viviani Viero

Hospital Universitário de Santa Maria - HUSM - Santa Maria (RS) - Brasil

Silvani Vargas Vieira

Universidade Federal de Santa Maria - UFSM - Santa Maria (RS) - Brasil

Nara Marilene Oliveira Girardon-Perlini

Universidade Federal de Santa Maria - UFSM - Santa Maria (RS) - Brasil

\section{RESUMO}

Objetivo: Verificar a efetividade das estratégias de marketing social e de acolhimento desenvolvidas em um hemocentro e, antes e após as ações realizadas, verificar a demanda reprimida de sangue e hemocomponentes em um hospital de referência. Métodos: Trata-se de estudo comparativo, com abordagem quantitativa, realizado em um hemocentro e no serviço de hemoterapia de um hospital de referência no município de Santa Maria, RS, Brasil, entre setembro e dezembro de 2015. As estratégias compreenderam ação de marketing social, realizada por meio do envio de cartas, e-mails e telefonemas às pessoas em condições de realizar uma nova doação, e o acolhimento, que consistiu em realizar a acolhida do usuário por meio de um álbum seriado. A partir do levantamento de informações do banco de dados, especificamente dos registros de transfusões solicitadas e efetivadas, comparou-se o número de doações efetivadas antes e após as ações ao quantitativo do ano anterior com base na estatística descritiva e teste $t$ de Student. Resultados: O número de doações em geral aumentou, principalmente no último mês em que ocorreram as ações $(\mathrm{p}=0,0397)$. Entretanto, a média de doações voluntárias de sangue total apresentou redução, passando de 237 doações/mês, em 2014, para 222 doações/mês, em 2015. A média de doações voluntárias de plaquetas por aférese aumentou de 11 doações/mês, em 2014, para 17 doações/mês, em 2015. Conclusão: As estratégias implementadas no período do estudo contribuíram para o aumento no número de doações de plaquetas por aférese, porém, com relação à doação de sangue total, não houve resultado positivo.

Descritores: Serviço de Hemoterapia; Sangue; Doadores de Sangue; Bancos de Sangue.

\section{ABSTRACT}

Objective: To assess the effectiveness of social marketing and user embracement strategies developed in a hemotherapy service and to assess the restrained demand for blood and blood components in a reference hospital before and after the actions. Methods: This is a quantitative comparative study carried out in a hemotherapy service and in a hemotherapy service of a reference hospital in the city of Santa Maria, Rio Grande do Sul, Brazil, between September and December of 2015. The strategies included social marketing through the sending of letters and e-mails and phone calls to people who could give blood again. User embracement consisted in welcoming users by showing them an information booklet. Data obtained from the database, particularly records of transfusions requested and performed, were used to compare the number of donations performed before and after the actions with data of the previous year using descriptive statistics and Student's t test. 
Results: The overall number of donations increased, especially in the last month when the interventions were performed ( $p=0.0397)$. The average number of voluntary blood donations decreased from 237 donations/month in 2014 to 222 donations/month in 2015. The average number of voluntary apheresis donations of platelets increased from 11 donations/month in 2014 to 17 donations/month in 2015. Conclusion: The strategies implemented during the research period were effective in increasing apheresis donation of platelets, but had no positive effects on whole-blood donation.

Descriptors: Hemotherapy Service; Blood; Blood Donors; Blood Banks.

\section{RESUMEN}

Objetivo: Verificar la efectividad de las estrategias de marketing social y de acogida desarrolladas en un hemocentro y verificar la demanda reprimida de sangre y hemocomponentes de un hospital de referencia antes y después de las acciones realizadas. Métodos: Se trata de un estudio comparativo de abordaje cuantitativo realizado en un hemocentro y en el servicio de hemoterapia de un hospital de referencia en el municipio de Santa María, RS, Brasil, entre septiembre y diciembre de 2015. Las estrategias fueron la acción de marketing social realizada a través del envío de cartas, correos electrónicos y llamadas a las personas con condiciones de realizar una nueva donación e la acogida que fue realizada al usuario a través de un álbum seriado. A partir de las informaciones del banco de datos, en especifico de los registros de las transfusiones solicitadas y efectuadas, se comparó el número de donaciones efectuadas antes y después de las acciones con el cuantitativo del año anterior basado en la estadística descriptiva y la prueba t de Student. Resultados: El número de donaciones ha aumentado en especial en el último mes de las acciones ( $p=0,0397)$. Sin embargo, la media de donaciones voluntarias de sangre total ha presentado una reducción de 237 donaciones/mes en 2014 para 222 donaciones/mes, en 2015. La media de donaciones voluntarias de plaquetas por aféresis ha aumentado de 11 donaciones/mes en 2014 para 17 donaciones/mes en 2015. Conclusión: Las estrategias implementadas en el periodo del estudio contribuyeron para el aumento del número de donaciones de plaquetas por aféresis, sin embargo, no hubo resultado positivo respecto la donación de sangre total.

Descriptores: Servicio de Hemoterapia; Sangre; Donantes de Sangre; Bancos de Sangre.

\section{INTRODUÇÃO}

A doação de sangue é um problema de interesse mundial, pois não há uma substância que possa, em sua totalidade, substituir o tecido sanguíneo ${ }^{(1)}$. Os hemocomponentes são utilizados há mais de 50 anos e são essenciais à prática médica. Sua coleta, acondicionamento e processamento envolvem etapas altamente especializadas, cuja produtividade depende da disponibilidade de doadores voluntários ${ }^{(2)}$. Este, portanto, pode ser considerado um dos maiores desafios enfrentados pelos hemocentros no mundo todo. Estudo realizado na Etiópia revelou que o conhecimento das pessoas acerca da doação de sangue voluntária é elevado, entretanto, a prática dessa atitude é baixa ${ }^{(3)}$. O Brasil, nesse contexto, enfrenta o mesmo desafio, ou seja, dificuldades para assegurar a obtenção e distribuição de sangue de forma segura e sustentável por meio da captação e da fidelização de doadores de sangue voluntários ${ }^{(4)}$. Este, portanto, pode ser considerado um dos maiores desafios enfrentados pelos hemocentros em todo o mundo.

Apesar da necessidade cada vez maior de doadores de sangue de primeira vez, uma maior frequência de doações de repetição possibilitaria a ampliação do atendimento à demanda crescente por hemocomponentes, a garantia de bolsas de sangue com maior segurança para o receptor e a redução do custo da coleta ${ }^{(5)}$.

O tipo de doação e o tipo de doador de sangue são classificados segundo os critérios adotados pelo Ministério da Saúde, na Portaria GM/MS n ${ }^{\circ} 158$, de 04 de fevereiro de 2016, que redefine o regulamento técnico de procedimentos hemoterápicos. Assim, a doação espontânea é definida como aquela realizada sem qualquer forma de benefício para o doador, e compreende a doação de uma unidade de sangue ou de um de seus componentes. A doação de reposição é a doação advinda do indivíduo que doa para atender a necessidade de um paciente específico, realizada por pessoas motivadas pelo próprio serviço, pela família ou por amigos, para repor o estoque de hemocomponentes do serviço de hemoterapia. A doação autóloga é do próprio paciente para seu uso exclusivo, enquanto a doação por aférese é aquela que possibilita a retirada de apenas uma das células do sangue $\operatorname{total}^{(6)}$.

A captação de doadores de sangue constitui-se como uma atividade voltada ao desenvolvimento de programas que orientem a população quanto à importância da doação voluntária ${ }^{()}$. Uma das formas para isso é a promoção social de conscientização e sensibilização das pessoas para a doação de sangue como ato de cidadania, solidariedade e preservação da vida humana ${ }^{(4)}$.

A divulgação sobre doação de sangue em meios de comunicação tem sido uma estratégia utilizada incessantemente para atingir principalmente a população de doadores voluntários de primeira vez, porém é imprescindível garantir que os que já doaram alguma vez tornem-se doadores voluntários para que sejam mantidos os estoques mínimos de sangue e hemocomponentes nos hemocentros ${ }^{(4)}$.

Nesta perspectiva, algumas estratégias específicas de captação são passíveis de serem realizadas com o intuito de alcançar esse público. O marketing social corresponde à utilização de um sistema de aviso/convocação por meio do recrutamento 
telefônico, envio de correspondência ou internet ${ }^{(7,8)}$. Outro recurso utilizado, não somente em hemocentros, mas também em unidades básicas de saúde, é o acolhimento, um tipo de estratégia institucional, considerado uma ferramenta eficaz e que pode trazer bom atendimento aos doadores e favorecer a fidelização, por permitir a potencialização do encontro entre o usuário, o profissional e o serviço ${ }^{(9)}$. Essas iniciativas necessitam da capacitação e da boa vontade dos profissionais ${ }^{(1)}$, e estudos têm evidenciado resultados favoráveis quando estratégias dessa natureza têm sido utilizadas na captação de doadores de sangue ${ }^{(1,7)}$.

Diante desse contexto, a avaliação da efetividade das ações empreendidas faz-se relevante para determinar a escolha de políticas a serem implementadas nos hemocentros e nos bancos de sangue, pois pode identificar aquelas capazes de potencializar a doação de sangue e corroborar com a criação de estratégias que objetivem a melhoria contínua desse processo. Ademais, proposições que apresentam alternativas para solucionar problemas relacionados à falta de sangue nos hospitais configuram-se como uma estratégia de promoção da saúde, uma vez que pode ser entendida, de forma ampliada, como um modo de pensar e de operar, relacionando-se a outras políticas e tecnologias desenvolvidas no sistema de saúde que contribuem para a construção de ações para responder às necessidades sociais em saúde ${ }^{(10)}$.

Assim, fundamentados no pressuposto de que ações de acolhimento e de marketing social poderiam contribuir para aumentar o número de doadores de sangue, repercutindo nos serviços de saúde, buscou-se implementá-las no cotidiano de um hemocentro. Dessa forma, o presente estudo teve como objetivo verificar a efetividade das estratégias de marketing social e de acolhimento desenvolvidas em um hemocentro e, antes e após as ações realizadas, verificar a demanda reprimida de sangue e hemocomponentes em um hospital de referência.

\section{MÉTODOS}

Trata-se de estudo de caráter comparativo, de abordagem quantitativa, realizado em um hemocentro público e em um serviço de hemoterapia de um hospital público de referência, na cidade de Santa Maria, Rio Grande do Sul, Brasil, realizado no período entre setembro e dezembro de 2015. O evento de comparação refere-se ao número de doações efetivadas no hemocentro e ao número de transfusões de hemocomponentes no hospital, antes e após as ações implementadas, compreendendo as informações de 5.049 doadores e registros de 5.376 unidades de plaquetas solicitadas e transfundidas, comparadas ao ano anterior.

Como critérios de inclusão, no hemocentro, definiram-se os dados efetivos de todos os doadores dos três meses de cada ano, 2014 e 2015, sendo excluídas do estudo as doações autólogas de sangue total. No serviço de hemoterapia do hospital, como critérios de inclusão, coletaram-se os dados de transfusões de plaquetas e excluíram-se os de transfusão de outros hemocomponentes. Os instrumentos utilizados para a coleta de dados foram dois formulários distintos, um para o hemocentro e outro para o serviço de hemoterapia do hospital, elaborados exclusivamente para o presente estudo.

$\mathrm{O}$ estudo realizado integra um projeto guarda-chuva ${ }^{(11)}$, que buscou desenvolver e avaliar ações de acolhimento e de marketing social em hemocentro. No acolhimento, a atividade consistiu em realizar a acolhida do usuário utilizando um álbum seriado explicativo sobre o processo de doação de sangue e de hemocomponentes. Dessa forma, enquanto o doador aguardava ser chamado para iniciar a triagem, era convidado à participar. A ação de marketing social realizou-se por meio do envio de cartas, e-mails e telefonemas às pessoas em condições de realizar uma nova doação (três meses para mulheres e dois meses para homens).

Para a coleta de dados, como forma de avaliar a efetividade das ações desenvolvidas, o presente estudo buscou comparar informações obtidas na base de dados eletrônica do hemocentro, antes e após a realização das estratégias. Para verificar o impacto no âmbito hospitalar, foram coletados dados em registros impressos do serviço de hemoterapia referentes aos meses de realização das ações (2015) e aos meses do ano anterior (2014).

Para análise dos dados obtidos nos dois locais do estudo, inicialmente, organizaram-se os dados em planilha do programa Excel e sua exportação para o programa SPSS, versão 23.0, sendo analisados com base na estatística descritiva. Para comparação dos dados, utilizou-se o teste t de Student e nível de significância de 0,05 .

O estudo recebeu aprovação do Comitê de Ética em Pesquisa da Universidade Federal de Santa Maria, sob parecer $n^{\circ}$ 1.189.482.

\section{RESULTADOS}

Dentre os 2.830 candidatos à doação nos meses de setembro, outubro e novembro de 2014, o total de rejeições na triagem clínica foi, em média, de $12,9 \%$ ( $\mathrm{n}=121)$ por mês. Do total de 2.863 candidatos à doação no mesmo período de 2015, o total de rejeições foi de $9,7 \%(\mathrm{n}=93)$ por mês.

A média de indivíduos aptos à doação de sangue e hemocomponentes foi de aproximadamente 820 doadores/mês em 2014 e 860 doadores/mês em 2015, havendo um aumento no número de doações no último ano. É possível notar que, nos dois primeiros meses de desenvolvimento das ações (setembro e outubro), houve similaridade no número de doadores em comparação ao ano anterior. No mês de novembro, comparando com o mesmo mês do ano anterior, identifica-se que houve um aumento estatisticamente significativo $(\mathrm{p}=0,0397)$ no número de doações, pois passaram de $34,56 \%(\mathrm{n}=852)$ para $37,34 \%$ $(\mathrm{n}=965)$ (figura 1). 


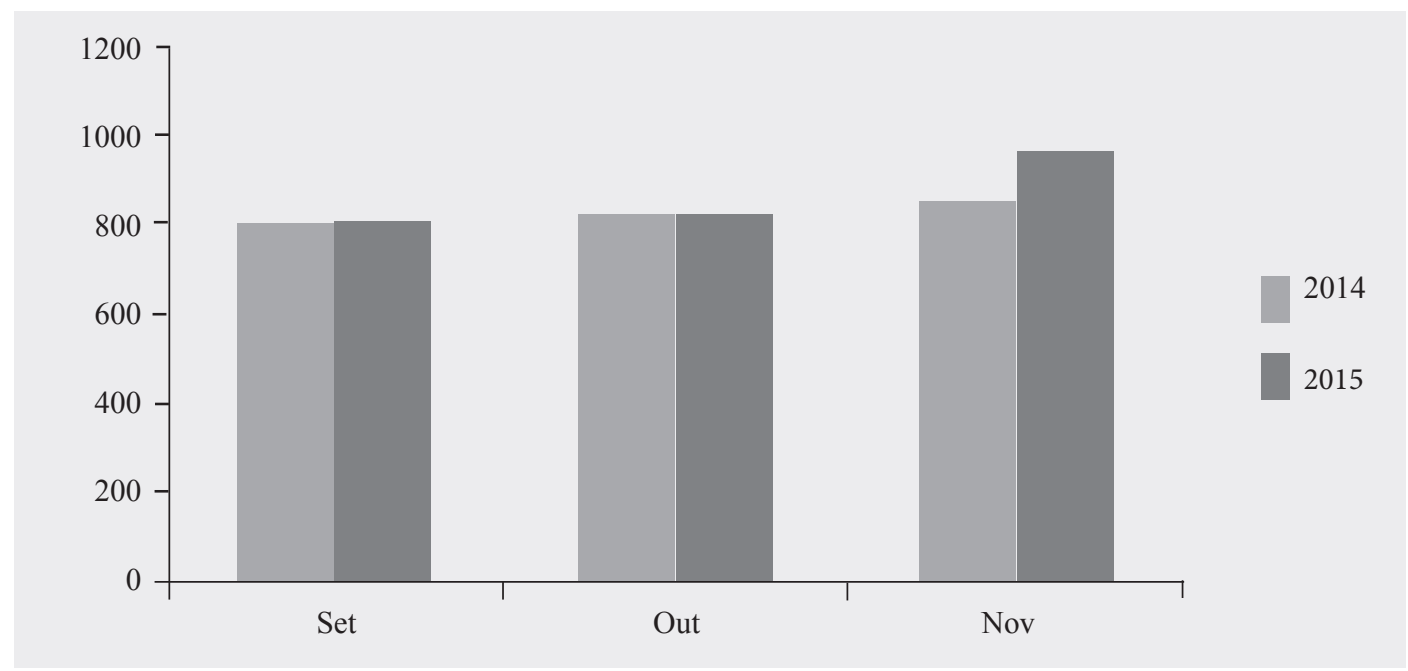

Figura 1 - Doações de sangue total e plaquetas por aférese nos meses de setembro, outubro e novembro de 2015 (meses das ações) comparados aos mesmos meses de 2014. Santa Maria, RS, 2015.

As doações de sangue total foram realizadas por doadores voluntários, doadores de reposição e doadores em campanhas realizadas nos municípios da região. As doações de plaquetas por aférese ocorreram apenas por doadores voluntários e de reposição. Na figura 2 estão apresentadas as quantidades, por tipo de doação, em cada mês/ano.

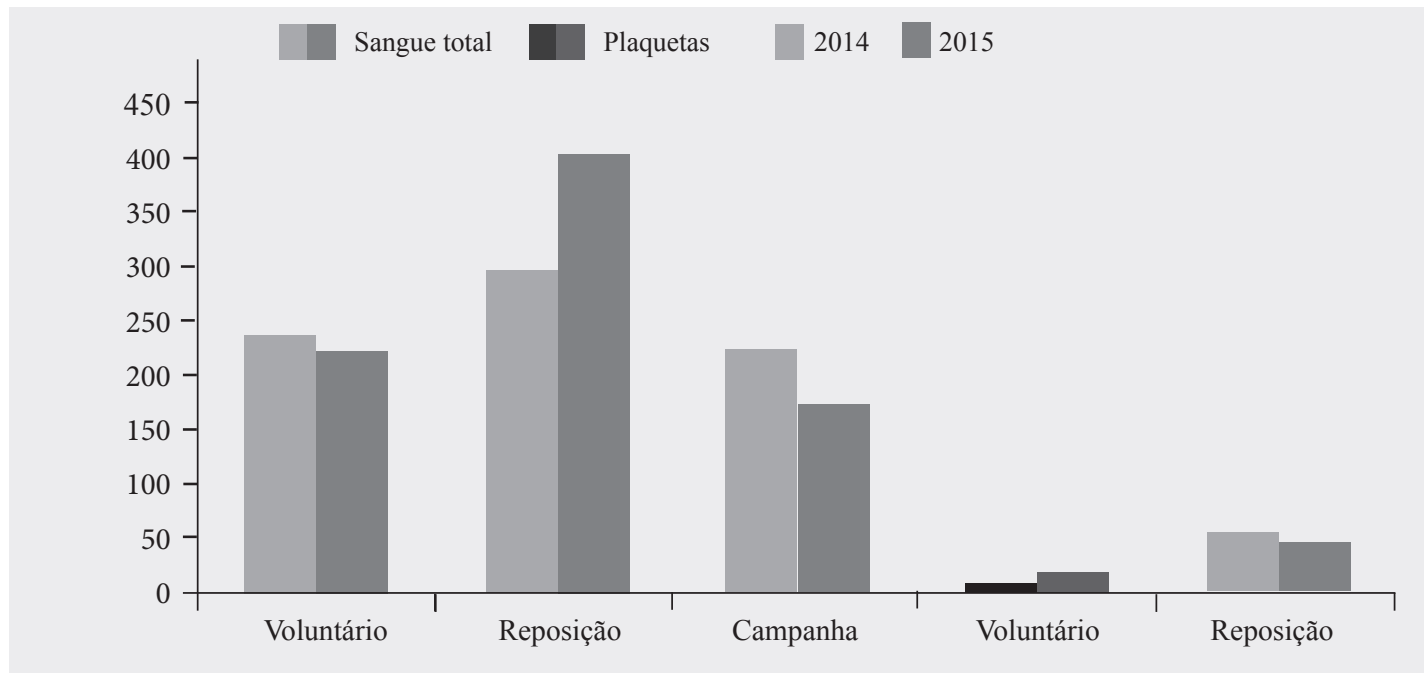

Figura 2 - Tipos de doação para sangue total e plaquetas por aférese antes e após as ações de marketing social. Santa Maria, RS, 2015 .

É possível perceber que a média do número de doações de sangue total por reposição aumentou em 2015. Essa doação, que é feita dirigida a um paciente específico, representou um aumento de $36,4 \%(\mathrm{n}=107)(\mathrm{p}=0,0552)$ quando comparada ao ano anterior. Em contrapartida, as doações voluntárias e por campanha diminuíram em 6,3\% $(n=15)(p=0,021)$ e 23,1\% $(n=52)$ $(\mathrm{p}=0,580)$ respectivamente.

No contexto hospitalar, no que diz respeito à demanda por sangue e hemocomponentes, são feitas transfusões de hemácias, plaquetas, plasma fresco e, em menor quantidade, de crioprecipitado. Pelo fato de a maioria dos hemocomponentes ter sido suprida, com exceção das plaquetas, procedeu-se uma busca específica desse hemocomponente para identificar qual é a demanda do local.

Destaca-se que a média de demanda por unidades de plaquetas, no período relativo ao ano de 2014, foi de 940 unidades/ mês, enquanto no ano de 2015 correspondeu a 852 unidades/mês. Os dados obtidos indicam que a demanda atendida de plaquetas equivale a 774 unidades/mês em 2014 e 786 unidades/mês em 2015 ( $\mathrm{p}=0,6143)$. Assim, a demanda reprimida, que equivale às unidades de plaquetas solicitadas e que não puderam ser atendidas por falta no estoque, reduziu significativamente, pois, em 2014, foi de 166 unidades/mês e, em 2015, perfez um total de 66 unidades/mês ( $p<0,0001$ ) (figura 3). 


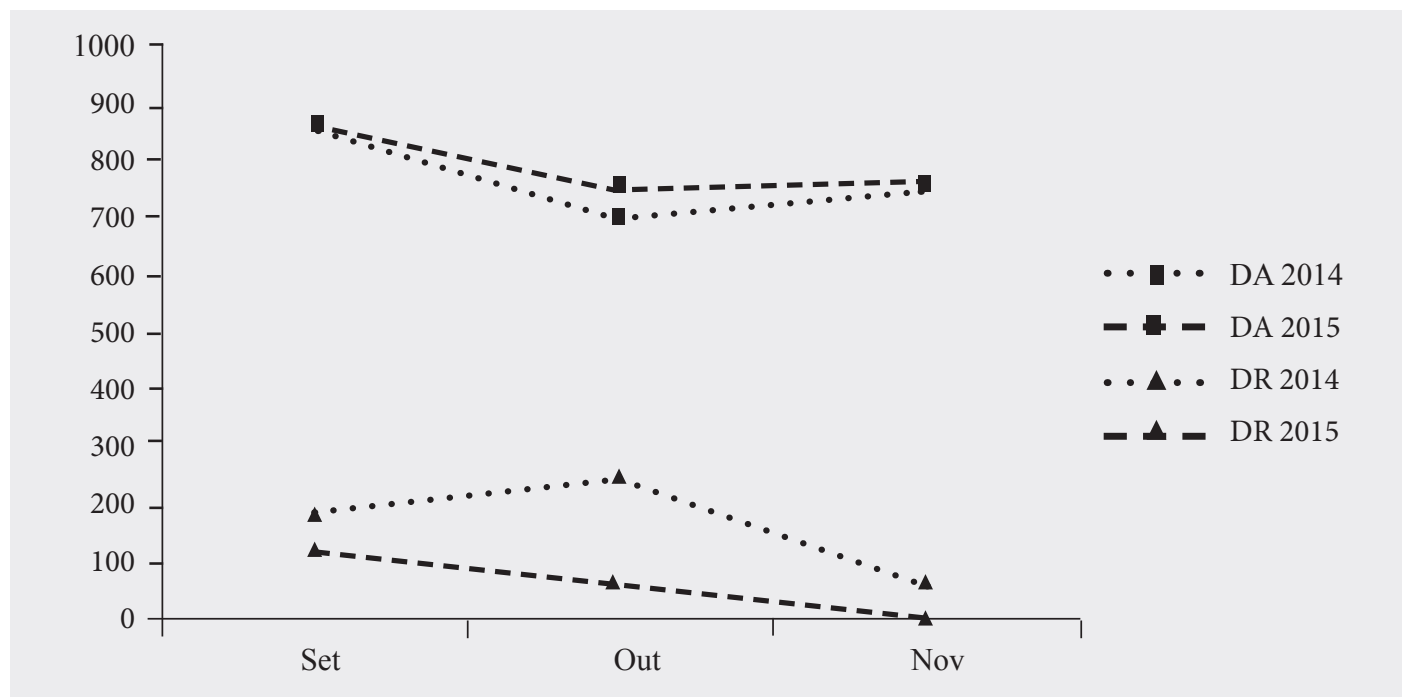

Figura 3 - Demanda de plaquetas no Hospital Universitário antes e após as ações de marketing social realizadas. Santa Maria, RS, 2015.

DR: Demanda atendida; DR: Demanda reprimida

\section{DISCUSSÃO}

A triagem no momento inicial do processo de doação de sangue é imprescindível para a seleção do indivíduo disposto a fazer sua doação, porém, nem sempre as pessoas estão aptas a realizarem. No presente estudo, no Hemocentro de Santa Maria a média de indivíduos inaptos à doação foi de 9,7\% em 2015 e 12,8\% em 2014. Em estudo realizado no Hemocentro de Campinas, de um total de 87.773 potenciais doadores, $24 \%$ foram considerados inaptos ${ }^{(12)}$. Em outro estudo foi possível perceber maior semelhança com os resultados do presente estudo, uma vez que $11,9 \%$ dos indivíduos também estavam inaptos para doar ${ }^{(13)}$. Os motivos da inaptidão são diversos, entre os quais está a falta de informação sobre os requisitos para doação de sangue, que determinam ou não a possibilidade de realizar a doação. Nota-se ainda, no presente estudo, que houve redução no número de pessoas inaptas à doação de um ano para o outro, o que pode estar relacionado ao conhecimento das pessoas sobre o processo de doação e aos critérios que as impedem.

É necessário que a população participe ativamente do processo de doação de sangue de forma responsável e consciente, o que é possível por meio de ações educativas e de mobilização social que visam a garantia da qualidade e quantidade adequada de sangue e hemocomponentes ${ }^{(14)}$. Dessa forma, quanto maior o número de candidatos esclarecidos sobre os requisitos necessários para doação, maiores serão as taxas de aptidão no momento da triagem clínica. Sugere-se que esse aumento possa estar relacionado principalmente às ações de marketing desenvolvidas em hemocentros para aumentar a captação de doadores. Essas ações, no presente estudo, deram-se por meio de envio de cartas, e-mails e por telefonemas àquelas pessoas em que o prazo para realizar uma nova doação já havia vencido (três meses para mulheres e dois meses para homens).

Nos meses de setembro e outubro, em que se realizaram as ações da atual pesquisa, mas não foram observadas alterações nos números de doadores, infere-se a possibilidade de ser decorrente do método utilizado para avaliar se as pessoas contatadas pelas ações de sensibilização realizaram a doação de sangue, pois a avaliação ocorreu muito próxima das ações. Convém destacar que o marketing social no processo de coleta de sangue é tido como uma ferramenta adicional de trabalho ${ }^{(15)}$, e, além do mais, é importante para a captação de doadores, sendo necessária a utilização de estratégias consistentes para tornar a doação de sangue parte de hábitos e valores da população ${ }^{(1)}$.

As ações de acolhimento na sala de espera, especificamente, estão direcionadas à fidelização do doador, que representa a possibilidade de torná-lo doador de repetição, ou seja, aquele que regular e espontaneamente comparece ao hemocentro para doar sangue ao menos duas vezes ao ano ${ }^{(6)}$. Considerando que o hemocentro de Santa Maria não dispõe de um profissional que possa realizar regularmente atividades de acolhimento na sala de espera e que esse processo de acolhida ao usuário deve ser efetuado por diversos profissionais em todas as fases do processo de doação, percebe-se, pelos resultados obtidos no presente estudo, que essa medida tem sido pouco eficaz para a fidelização, embora seja válida como prática de estímulo à doação.

É necessário ressaltar que a melhoria e a humanização dos processos de acolhimento são fundamentais para conquistar o doador, por isso é imprescindível que haja um treinamento adequado dos profissionais envolvidos nesse processo ${ }^{(16)}$. Além disso, a escuta ativa realizada pelo profissional propicia oportunidade para o doador expressar o que sabe, pensa e sente, tornando um momento de socialização do conhecimento sobre as suas necessidades e de como satisfazê-las ${ }^{(17)}$. 
Embora as ações de acolhimento e marketing social apresentem resultados promissores na captação de doadores, somente podem ser avaliadas passado o intervalo de tempo necessário para uma nova doação, que é, para os homens, no mínimo de dois meses, não excedendo quatro doações por ano; e, para as mulheres, no mínimo de três meses, não excedendo três doações por ano $^{(18)}$.

A doação por reposição, conforme classificado pelo Ministério da Saúde ${ }^{(6)}$, é quando um indivíduo doa para atender as necessidades de um paciente. No Hemocentro de Santa Maria, esse tipo de doação representou 42,4\% das doações de sangue e plaquetas em 2014, subindo para 52,2\% em 2015. Em uma pesquisa realizada em três centros de doação de sangue do Brasil ${ }^{(19)}$, foram encontrados resultados entre $24,3 \%$ e $53,6 \%$ de doações realizadas por reposição.

Quanto à doação de plaquetas por aférese, na atual pesquisa pode-se perceber um aumento significativo $(p=0,033)$ no número de doadores voluntários $(54,5 \%)$ quando comparado ao ano anterior, e uma pequena redução $(\mathrm{p}=0,336)$ das doações de reposição $(9,2 \%)$. Com base nos dados coletados no banco de dados no setor de hemoterapia do Hospital Universitário, há aproximadamente um ano não houve dificuldade em suprir a demanda de sangue com relação à maioria dos hemocomponentes, exceto de plaquetas. Em vista disso, considera-se positivo o aumento de doadores voluntários de plaquetas por aférese, já que se sabe da importância do aumento do número desses para não depender de doadores de reposição. A doação por reposição causa angústia aos familiares dos pacientes necessitados, os quais geralmente são responsabilizados pela busca de doadores. Essa necessidade geralmente se torna um processo "árduo" para a família, principalmente pelo fato de que a transfusão é fator fundamental para a continuidade do tratamento ${ }^{(20)}$.

Além disso, a população ainda desconhece a necessidade da transfusão de sangue causada por diversas situações de saúde, e pensa que é um problema fácil de ser resolvido pelos parentes, pelas instituições militares ou pelos profissionais da área da saúde ${ }^{(21)}$, por isso ações educativas e de sensibilização devem ser desenvolvidas, de forma que desperte a solidariedade da população. Em uma pesquisa internacional, realizada para investigar o motivo para a doação de sangue total, concluiu-se que as razões mais frequentemente relatadas para doar sangue pela primeira vez foram " influência de um amigo" (47,2\% dos doadores) e marketing (23,5\% de doadores). Com relação a continuar a doar sangue, o altruísmo e a responsabilidade social foram os motivos mais frequentes das respostas, com $68,4 \%$ e $16,0 \%$, respectivamente. Ainda na mesma pesquisa, percebeu-se outro dado interessante com relação ao obstáculo mais comumente relatado para se tornar um doador de sangue regular, sendo encontrado, em primeiro lugar, a " preguiça" (19,1\%), seguido por "medo de agulhas" $(10,5 \%)^{(22)}$.

A falta de plaquetas para serem transfundidas é uma realidade que angustia os profissionais da saúde e, na maioria das vezes, causa transtorno aos familiares dos pacientes que dependem desse componente sanguíneo ${ }^{(20)}$. Nota-se que, apesar de haver um aumento na demanda atendida de plaquetas de um ano para o outro, no presente estudo, este valor não foi significativo $(\mathrm{p}=0,6143)$, mas a demanda reprimida obteve uma redução considerável $(\mathrm{p}<0,0001)$ em 2015. Com base nisso, vale ressaltar que a demanda atendida foi maior, assim como a solicitada, motivo pelo qual a demanda reprimida apresentou redução. A necessidade desse componente sanguíneo se dá em pacientes com trombocitopenia, sendo utilizado na prevenção de hemorragia espontânea, que pode ocorrer em pacientes que apresentam contagens de plaquetas abaixo de $10.000 / \mathrm{mm}^{3}$, realidade de pacientes hemato-oncológicos em tratamento quimioterápico ${ }^{(23)}$.

Embora dados estatísticos mundiais apontem que as doações de sangue não acompanham o aumento das transfusões ${ }^{(1)}$, os resultados do presente estudo demonstraram que, apesar do aumento da demanda por plaquetas, houve concomitantemente o aumento no atendimento aos pacientes que necessitaram desse hemocomponente, o que revela uma maior disponibilidade no estoque de sangue. Considera-se esse resultado favorável à implementação de terapêuticas que contribuam na melhoria da saúde dos pacientes tratados em um hospital referência para o tratamento de adultos e crianças com doenças hemato-oncológicas. Cabe destacar que estratégias como essas fazem parte do processo de promoção da saúde, formadas a partir da participação social, empoderamento e reorientação dos serviços de saúde, entre outros eixos de ação que visam provocar a mudança nas condições de vida das pessoas em torno de um determinante social ${ }^{(24)}$, nesse caso, a doação de sangue.

Com relação às limitações do atual estudo, destaca-se o tempo disponível para verificar a efetividade das estratégias de marketing social e de acolhimento, cuja avaliação foi muito próxima ao término das mesmas, não possibilitando um período maior para o retorno das pessoas que participaram das atividades, bem como daquelas que teriam que aguardar o intervalo necessário para nova doação. Cabe ressaltar que estudos que objetivam verificar a efetividade desses tipos de estratégias necessitam de um acompanhamento por maior período de tempo, ao menos respeitando o intervalo entre uma doação e outra. Considerando-se que a informação é a base para o conhecimento, não poderia ser diferente com relação à doação de sangue, sendo válido reforçar a sua importância aos profissionais envolvidos no processo de doação e, mais do que isso, à população, disseminando a educação do ato humano de doar sangue.

\section{CONCLUSÃO}

É possível afirmar que as estratégias implementadas no período deste estudo foram contributivas para o aumento no número de doações de plaquetas por aférese, porém, com relação à doação de sangue total, não houve resultado positivo. Ações voltadas à captação de doadores de sangue precisam ser realizadas constantemente, e não isoladamente, sendo necessário haver a associação de mais de uma estratégia, pois assim, cada qual atinge um determinado público, com o intuito de alcançar o 
máximo de pessoas possíveis para manter os estoques de sangue e hemocomponentes do hemocentro sempre em quantidades adequadas.

\section{REFERÊNCIAS}

1. Rodrigues RSM, Reibnitz KS. Estratégias de captação de doadores de sangue: uma revisão integrativa da literatura. Texto \& Contexto Enferm. 2011;20(2):384-91.

2. Sekine L, Wirth LF, Faulhaber GAM, Seligman BGS. Análise do perfil de solicitações para transfusão de hemocomponentes no Hospital de Clínicas de Porto Alegre no ano de 2005. Rev Bras Hematol Hemoter. 2008;30(3):208-12.

3. Melku M, Terefe B, Asrie F, Enawgaw B, Melak T, Tsegay YG, et al. Knowledge, attitude, and practice of adult population towards blood donation in Gondar Town, Northwest Ethiopia: a community based cross-sectional study. J Blood Transfus. 2016;2016:1-10.

4. Lopes ECS, Guedes CCP, Aguiar BGC. Estratégias para a captação de doadores de sangue difundidas na literatura. Rev Acred. 2012;2(4):104-21.

5. Ludwig ST, Rodrigues ACM. Doação de sangue: uma visão de marketing. Cad Saúde Pública. 2005;21(3):932-9.

6. Ministério da Saúde (BR), Secretaria de Gestão do Trabalho e da Educação na Saúde, Departamento de Gestão do Trabalho na Saúde. Técnico em hemoterapia: livro texto. Brasília: Ministério da Saúde; 2013.

7. Giacomini L, Lunardi WD Filho. Estratégias para fidelização de doadores de sangue voluntários e habituais. Acta Paul Enferm. 2010;23(1):65-72.

8. Gallego M, Muñoz L, Cortés A. Caracteristicas socioculturales de los donantes y no donantes de sangre en Colombia. Colomb Méd. 2000;31(3):99-109.

9. Oliveira JR, Albuquerque MCS, Brêda MZ, Barros LA, Lisbôa GLP. Concepções e práticas de acolhimento apresentadas pela enfermagem no contexto da atenção básica à saúde. Rev Enferm UFPE online. 2015;9(Supl 10):1545-55.

10. Ministério da Saúde (BR), Secretaria de Vigilância em Saúde, Secretaria de Atenção à Saúde. Política Nacional de Promoção da Saúde. $3^{\mathrm{a}}$ ed. Brasília: Ministério da Saúde; 2010.

11. Santos CF, Carlesso L, Guimarães RFS, Silva SL. Captação de doadores voluntários de sangue e hemocomponentes: contribuições de uma equipe multiprofissional [monografia]. Santa Maria: Universidade Federal de Santa Maria; 2016.

12. Lieber SR, Oliveira BB, Baungartner JE. Principais causas de inaptidão clínica dos doadores de sangue do hemocentro de campinas [acesso em 2016 Jan 5]. IV Simpósio de Profissionais da Unicamp, Campinas; 2012 Nov 6-7. Disponível em: http://www.sbu.unicamp.br/seer/ojs/index.php/simtec/article/view/7441/2984

13. Menezes AG, Sousa CV, Cunha GR. Motivational aspects in blood donation: an analysis under the perspective of marketing theories. Bus Manag Review. 2015;4(5):330-41.

14. Moura AS, Moreira CT, Machado CA, Vasconcelos JA Neto, Machado MFAS. Doador de sangue habitual e fidelizado: fatores motivacionais de adesão ao programa. Rev Bras Promoç Saúde. 2006;19(2):61-7.

15. Aldamiz-Echevarria C, Aguirre-Garcia MS. A behavior model for blood donors and marketing strategies to retain and attract them. Rev Latinoam Enferm. 2014;22(3):467-75.

16. Capra MS. Fidelização de doadores de sangue voluntários e habituais: uma prática de educação em saúde [dissertação]. Porto Alegre: Universidade do Vale do Rio dos Sinos, 2013.

17. Araújo FMR, Feliciano KVO, Mendes MFM. Aceitabilidade de doadores de sangue no hemocentro público do Recife, Brasil. Ciênc Saúde Coletiva. 2011;16(12):4823-32.

18. Ministério da Saúde (BR). Portaria $\mathrm{n}^{\circ} 2.712$, de 12 de novembro de 2013. Redefine o regulamento técnico de procedimentos hemoterápicos. Brasília: Ministério da Saúde, 2013.

19. Carneiro-Proietti AB, Sabino EC, Sampaio D, Proietti FA, Gonçalez TT, Oliveira CDL, et al. Demographic profile of blood donors at three major Brazilian blood centers: results from the International REDS-II study, 2007 to 2008. Transfusion. 2010;50(4):918-25.

20. Neckel VC. 'A gente acha que nunca vai precisar.' A vivência de familiares e usuários do serviço de hematologia-oncologia acerca do processo de captação de doadores de sangue no Hospital Universitário de Santa Maria [monografia]. Santa Maria: Universidade Federal de Santa Maria; 2014. 
21. Reginato MARM, Andrade CC. Captação de doadores: uma prática de educação em saúde e de mobilização social vivenciada no hemonúcleo de Guarapuava - PR. Unicentro Rev Eletrônica Lato Sensu [Internet]. 2008 [acesso em 2016 Jan 5];5:1-24. Disponível em: http://www.escoladegestao.pr.gov.br/arquivos/File/anais/painel_saude/captacao_de_doadores.pdf

22. Sojka EN, Sojka P. The blood donation experience: self-reported motives and obstacles for donations blood. Vox Sang. 2008;94(1):56-63.

23. Razouk FH, Reiche EMV. Caracterização, produção e indicação clínica dos principais hemocomponentes. Rev Bras Hematol Hemoter [online]. 2004;26(2):126-34.

24. Maeyama MA, Jasper CH, Nilson LG, Dolny LL, Cutolo LRA. Promoção da saúde como tecnologia para transformação social. Rev Bras Tecnol Sociais. 2015;2(2):129-43.

\section{Endereço para correspondência:}

Leticia Carlesso

Universidade Federal de Santa Maria - UFSM

Av. Roraima, 1000 - prédio 26/ sala 1339

Bairro: Camobi - Cidade Universitária

CEP: 97105-900 - Santa Maria - RS - Brasil

E-mail: leticarlesso@gmail.com 